Article

\title{
Geodesic Mappings of Spaces with Affine Connections onto Generalized Symmetric and Ricci-Symmetric Spaces
}

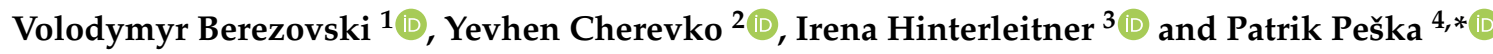 \\ 1 Department of Mathematics and Physics, Uman National University of Horticulture, 20300 Uman, Ukraine; \\ Berez.Volod@gmail.com \\ 2 Department of Physics and Mathematics Sciences, Odesa National Academy of Food Technologies, \\ 65039 Odesa, Ukraine; Cherevko@usa.com \\ 3 Institute of Mathematics and Descriptive Geometry, Brno University of Technology, 60200 Brno, \\ Czech Republic; Hinterleitner.Irena@seznam.cz \\ 4 Department of Algebra and Geometry, Palacký University Olomouc, 77147 Olomouc, Czech Republic \\ * Correspondence: Patrik.Peska@upol.cz
}

Received: 28 July 2020; Accepted: 8 September 2020; Published: 11 September 2020

\begin{abstract}
In the paper, we consider geodesic mappings of spaces with an affine connections onto generalized symmetric and Ricci-symmetric spaces. In particular, we studied in detail geodesic mappings of spaces with an affine connections onto 2-, 3-, and $m$ - (Ricci-) symmetric spaces. These spaces play an important role in the General Theory of Relativity. The main results we obtained were generalized to a case of geodesic mappings of spaces with an affine connection onto (Ricci-) symmetric spaces. The main equations of the mappings were obtained as closed mixed systems of PDEs of the Cauchy type in covariant form. For the systems, we have found the maximum number of essential parameters which the solutions depend on. Any $m$ - (Ricci-) symmetric spaces $(m \geq 1)$ are geodesically mapped onto many spaces with an affine connection. We can call these spaces projectivelly m- (Ricci-) symmetric spaces and for them there exist above-mentioned nontrivial solutions.
\end{abstract}

Keywords: geodesic mapping; space with an affine connection; $m$-symmetric space; $m$-Ricci-symmetric space

MSC: 53B05; 53B50; 35M10

\section{Introduction}

The paper is devoted to further study of the theory of geodesic mappings of affinely connected spaces. The theory goes back to the paper [1] by T. Levi-Civita in which the problem on the search for Riemannian spaces with common geodesics was stated and solved in a special coordinate system. We note the remarkable fact that this problem is related to the study of equations of dynamics of mechanical systems.

The theory of geodesic mappings was developed by T.Y.Thomas, J.M. Thomas, H. Weyl, P.A. Shirokov, A.S. Solodovnikov, N.S. Sinyukov, A.V. Aminova, J. Mikeš, and others [2-17].

The spaces with covariantly constant curvature tensor (symmetric spaces) were considered in 1920 by P.A. Shirokov [5], E. Cartan [18], and A. Lichnerowicz [19], and with covariantly parallel curvature tensor (recurrent spaces) [20]. The study of symmetric and recurrent spaces is an extensive part of differential geometry and its applications. 
It is well-known that the spaces of constant curvature are symmetric and for them E. Beltrami proved that they admit nontrivial geodesic mappings. In 1954, N.S. Sinyukov [7] began to study geodesic mappings of symmetric, recurrent, and semisymmetric spaces with equiaffine connection onto (pseudo-) Riemannian spaces. Continuation of these studies we can find in the works [21-25], V. Fomin [26], I. Hinterleitner, and J. Mikeš [27]. The above-mentioned results have a negative character in the sense that the space of non-constant curvature does not admit nontrivial geodesic mappings. T. Sakaguchi [28] and V. Domashev, J. Mikeš [29] studied similar tasks for holomorphically projective mappings. In the paper by V.Berezovski et al. [30], it is possible to find the generalized case of geodesic mappings of symmetric spaces.

Later, there were studied more generalized spaces than symmetric and recurrent ones. Generalized symmetric and recurrent spaces were comprehensively studied by V.R. Kaigorodov [31-36] from the point of view of the General Theory of Relativity. The paper [35] is a detailed analysis of this issue; it contains 97 citations. In another direction, symmetric spaces are generalized, for example, in works [37,38].

For geodesic mappings of generalized symmetric and recurrent spaces, such problems were solved by J. Mikeš, V.S. Sobchuk, and others [21-27,38-48]. There are many works devoted to issues of the theory of geodesic mappings, for example [49-58].

The above-mentioned results with proofs are in the works $[12,13,15,17]$.

In our work, we continue the study of geodesic mappings of generalized symmetric spaces with an affine connection.

We suppose that all spaces under consideration are spaces with an affine connections without torsion. In addition, we assume that all geometric objects under consideration are not only continuous but also sufficiently smooth.

\section{Basic Concepts of the Theory of Geodesic Mappings of Spaces with Affine Connections}

A diffeomorphism between two spaces with an affine connections is an one-to-one differentiable mapping, and the inverse mapping is differentiable too. Among diffeomophisms, there are very important ones which are referred to as geodesic mappings.

Let us suppose that a space $A_{n}$ with an affine connection $\nabla$ admits a diffeomorphism $f$ onto another space $\bar{A}_{n}$ with an affine connection $\bar{\nabla}$ and locally the spaces are referred to a common coordinate system $x, x=\left(x^{1}, x^{2}, \ldots, x^{n}\right)$.

Assume $P=\bar{\nabla}-\nabla$ and a in local coordinate system

$$
P_{i j}^{h}(x)=\bar{\Gamma}_{i j}^{h}(x)-\Gamma_{i j}^{h}(x),
$$

where $\Gamma_{i j}^{h}(x)$ and $\bar{\Gamma}_{i j}^{h}(x)$ are components of affine connections $\nabla$ and $\bar{\nabla}$ of the spaces $A_{n}$ and $\bar{A}_{n}$, respectively, expressed with respect to the common coordinate system $x$. The tensor $P$ is called a deformation tensor of the connections $\nabla$ and $\bar{\nabla}$ with respect to the mapping $f$.

A curve $\ell: x=x(t)$ in a space $A_{n}$ with an affine connection $\nabla$ is a geodesic when its tangent vector $\lambda(t)=d x(t) / d t$ satisfies the equations

$$
\nabla_{t} \lambda=\rho(t) \cdot \lambda,
$$

where $\nabla_{t}$ denotes the covariant derivative along $\ell$ and $\rho(t)$ is some function.

A diffeomorphism $f: A_{n} \rightarrow \bar{A}_{n}$ is an geodesic mapping if any geodesic of $A_{n}$ is mapped under $f$ onto a geodesic in $\bar{A}_{n}$.

The most known equations of geodesic mappings are the Levi-Civita equations. He has obtained the equations for Riemannian spaces [1]. For the case of affinely connected spaces, the equation was later obtained by H. Weyl [4]. In the paper [59], the authors present alternative proofs for the Levi-Civita equation. 
It is known [1-17] that the mapping $f$ of a space $A_{n}$ onto a space $\bar{A}_{n}$ is geodesic, if and only if in a common coordinate system $x$ the deformation tensor has the form (the Levi-Civita equation)

$$
P_{i j}^{h}(x)=\psi_{i}(x) \delta_{j}^{h}+\psi_{j}(x) \delta_{i}^{h},
$$

where $\delta_{i}^{h}$ is the Kronecker delta and $\psi_{i}$ is a covector.

A geodesic mapping is called non-trivial if $\psi_{i} \not \equiv 0$. It is obvious that any space $A_{n}$ with an affine connection admits a non-trivial geodesic mapping onto space $\bar{A}_{n}$ with an affine connection. However, generally speaking, the similar statement would be wrong for geodesic mappings between Riemannian spaces. In particular, there are classes of Riemannian spaces which do not admit non-trivial geodesic mappings onto other Riemannian spaces.

In the general case, the main equations of geodesic mappings of spaces with an affine connections can not be reduced to closed systems of differential equations of Cauchy-type since the general solutions depend on $n$ arbitrary functions $\psi_{i}(x)$.

N.S. Sinyukov [8,9] proved that the main equations for geodesic mappings of (pseudo-) Riemannian spaces are equivalent to some linear system of differential equations of Cauchy-type in covariant derivatives.

J. Mikeš and V. Berezovski [50] proved that the main equations for geodesic mapping of space with an affine connection onto a (pseudo-) Riemannian space can also be reduced to a closed system of PDE's of Cauchy type. In the case of geodesic mappings of an equiaffine space onto a (pseudo-) Riemannian space, the main equations are equivalent to some linear system of Cauchy-type in covariant derivatives. This property for all spaces with an affine connection follows from the results by J.M. Thomas [3], see $[15,16]$ that any space with an affine connection is projectivelly equivalent to an equiaffine space.

Refs. [46-48] were devoted to geodesic mappings of spaces with an affine connections onto Ricci-symmetric and 2-Ricci-symmetric spaces. The main equations for the mappings were also obtained as closed systems of PDE's of Cauchy type. A more detailed description of the theory of partial differential equations (PDE's) of the Cauchy type can be found in the books ([15] pp. 100-105) and ([17] pp. 130-134).

It is known $[7,12-17]$ that, in a common coordinate system $x$, respective to the mapping, the components of the Riemannian tensors $R_{i j k}^{h}$ and $\bar{R}_{i j k}^{h}$ of spaces with an affine connections $A_{n}$ and $\bar{A}_{n}$, respectively, are in the relation

$$
\bar{R}_{i j k}^{h}=R_{i j k}^{h}+P_{i k, j}^{h}-P_{i j, k}^{h}+P_{i k}^{\alpha} P_{j \alpha}^{h}-P_{i j}^{\alpha} P_{k \alpha}^{h} .
$$

Throughout the paper, the comma denotes the covariant derivative with respect to the connection $\nabla$ of the space $A_{n}$. Taking account of (2), from (3), we obtain

$$
\bar{R}_{i j k}^{h}=R_{i j k}^{h}-\delta_{j}^{h} \psi_{i, k}+\delta_{k}^{h} \psi_{i, j}-\delta_{i}^{h} \psi_{j, k}+\delta_{i}^{h} \psi_{k, j}+\delta_{j}^{h} \psi_{i} \psi_{k}-\delta_{k}^{h} \psi_{i} \psi_{j}
$$

Contracting the Equation (4) for $h$ and $k$, we get

$$
\bar{R}_{i j}=R_{i j}+n \psi_{i, j}-\psi_{j, i}+(1-n) \psi_{i} \psi_{j},
$$

where $R_{i j}$ and $\bar{R}_{i j}$ are the Ricci tensors of the spaces with an affine connections $A_{n}$ and $\bar{A}_{n}$, respectively.

From the Equation (5), it follows that

$$
\psi_{i, j}=\psi_{i} \psi_{j}+\frac{2}{n^{2}-1}\left(n \bar{R}_{i j}+\bar{R}_{j i}-\left(n R_{i j}+R_{j i}\right)\right) .
$$

In particular, Equation (6) was obtained in the papers [46-48]. 


\section{On $m$-Symmetric Spaces and Ricci $m$-Symmetric Spaces}

As we mentioned earlier, symmetric spaces were considered in 1920 by P.A.Shirokov [5], E. Cartan [18], and A. Lichnerowicz [19]. These spaces are characterized by covariantly constant curvature tensor, i.e., $\nabla R=0$. Their generalizations are recurrent spaces studied by H.S. Ruse [20] with covariantly parallel curvature tensor, $\nabla R=\varphi \circ R$. The spaces were generalized in many ways.

One of the most general generalizations are generalized m-recurrent $\left(D_{n}^{m}\right), m$-recurrent $\left(K_{n}^{m}\right)$ and $m$-symmetric $\left(S_{n}^{m}\right)$ spaces, which are in turn characterized by relations

$$
\begin{aligned}
& \nabla^{m} R=\Omega \circ R+\Omega^{(1)} \circ \nabla R+\Omega^{(2)} \circ \nabla^{2} R+\cdots+\Omega^{(m-1)} \circ \nabla^{m-1} R, \\
& \nabla^{m} R=\Omega \circ R, \\
& \nabla^{m} R=0,
\end{aligned}
$$

where $\nabla^{m}=\nabla\left(\nabla^{m-1}\right), \Omega(\neq 0), \Omega^{(1)}, \ldots, \Omega^{(m-1)}$ are tensors.

V.R. Kaigorodov [35] defined these spaces and studied them in detail.

The natural generalizations of these spaces are generalized m-Ricci-recurrent $\left(\operatorname{Ric} D_{n}^{m}\right)$, $m$-Ricci-recurrent $\left(\operatorname{RicK}_{n}^{m}\right)$, and m-Ricci-symmetric $\left(\operatorname{Ric} S_{n}^{m}\right)$ spaces, which are in turn characterized by relations

$$
\begin{aligned}
& \nabla^{m} R i c=\Omega \circ R i c+\Omega^{(1)} \circ \nabla R i c+\Omega^{(2)} \circ \nabla^{2} R i c+\cdots+\Omega^{(m-1)} \circ \nabla^{m-1} \text { Ric }, \\
& \nabla^{m} R i c=\Omega \circ R i c, \\
& \nabla^{m} \text { Ric }=0 .
\end{aligned}
$$

Our work is devoted to the study of the $m$-symmetric and $m$-Ricci symmetric spaces. Therefore, we present an example of four-dimensional pseudo-Riemannian $m$-symmetric spaces, which is not $\ell$-symmetric, $\ell=1,2, \ldots, m-1$, see ([35] p. 192):

$$
\left.d s^{2}=-d x^{2^{2}}-d x^{3^{2}}+2 d x^{4} \cdot\left[d x^{1}+\left(\alpha_{p q} x^{p} x^{q}+\beta_{p} x^{p}\right) d x^{4}\right)\right],
$$

where $\beta_{p}$ are function on $x^{4}$ and $\alpha_{p q}$ are polynoms

$$
\alpha_{p q}=\stackrel{(1)}{a}_{p q}\left(x^{4}\right)^{m-1}+\stackrel{(2)}{a}_{p q}\left(x^{4}\right)^{m-2}+\cdots+\stackrel{(m-1)}{a}_{p q} x^{4}+\stackrel{(m)}{a}_{p q}
$$

$\stackrel{(\ell)}{a}_{p q}(\ell=1,2, \ldots, m, p, q=2,3)$ are constants with $\stackrel{(1)}{a}_{p q} \not \equiv 0$.

We construct an example of 4-dimensional pseudo-Riemannian Ricci $m$-symmetric spaces which is not Ricci $\ell$-symmetric, $\ell=1,2, \ldots, m-1$. These spaces are with the above-mentioned metric with function $\beta_{p}$ of variable $x^{4}, \alpha_{p q}(p . q=2,3)$ are $m$ times differentiable function of $x^{4}$ and $\alpha_{22}+\alpha_{33}$ is the polynom

$$
\alpha_{22}+\alpha_{33}=\stackrel{(1)}{a}\left(x^{4}\right)^{m-1}+\stackrel{(2)}{a}\left(x^{4}\right)^{m-2}+\cdots+\stackrel{(m-1)}{a} x^{4}+\stackrel{(m)}{a},
$$

where $\stackrel{(\ell)}{a}(\ell=1,2, \ldots, m)$ are constants with $\stackrel{(1)}{a} \not \equiv 0$.

It is easy to construct more dimensional $m$-symmetric and $m$-Ricci symmetric spaces as product spaces of above-mentioned spaces and also trivial spaces which are e.g., spaces of constant curvature.

Recall the main results of geodesic mappings onto $m$-symmetric and Ricci $m$-symmetric spaces:

1. N.S. Sinyukov [7]: If equiaffine symmetric and recurrent spaces admit non-trivial geodesic mappings onto (pseudo-) Riemannian spaces $\bar{V}_{n}$ then $\bar{V}_{n}$ is the space of constant curvature.

2. V.V. Fomin [26]: If symmetric and recurrent spaces with infinity dimension admit non-trivial geodesic mappings onto (pseudo-) Riemannian spaces $\bar{V}_{n}$, then $\bar{V}_{n}$ is space of constant curvature. 
3. I. Hinterleitner and J. Mikeš [27]: If equiaffine symmetric and recurrent spaces admit non-trivial geodesic mappings onto (pseudo-) Weyl spaces $\bar{V}_{n}$, then $\bar{V}_{n}$ is space of constant curvature.

4. J. Mikeš [21,23]: If generalized recurrent spaces $D_{n}^{m}$ admit non-trivial geodesic mappings onto (pseudo-) Riemannian spaces $\bar{V}_{n}$, then $\bar{V}_{n}$ is space of constant curvature.

5. J.Mikeš [22]: If Ricci 2-symmetric spaces admit non-trivial geodesic mappings onto (pseudo-) Riemannian spaces $\bar{V}_{n}$, then $\bar{V}_{n}$ is Einstein space.

6. J. Mikeš, V.S. Sobchuk [39]: If Ricci 3-symmetric spaces admit non-trivial geodesic mappings onto (pseudo-) Riemannian spaces $\bar{V}_{n}$, then $\bar{V}_{n}$ is Einstein space.

7. V.S. Sobchuk [40]: If Ricci 4-symmetric spaces admit non-trivial geodesic mappings onto (pseudo-) Riemannian spaces $\bar{V}_{n}$, then $\bar{V}_{n}$ is Einstein space.

8. J. Mikeš [23,24]: If Ricci m-symmetric spaces admit non-trivial geodesic mappings onto (pseudo-) Riemannian spaces $\bar{V}_{n}$, then $\bar{V}_{n}$ is Einstein space.

A summary of these results and their proofs contain monographs [12-17].

The above results are "trivial" geodesic mappings in nature, i.e., under the above conditions, the spaces allow only trivial geodesic mapping. On the other hand, spaces with an affine connection, $m$-symmetric, and $m$-Ricci-symmetric spaces admit non trivial geodesic mappings onto affine connected spaces $\bar{A}_{n}$. Our article is devoted to these questions.

\section{Geodesic Mappings of Spaces with Affine Connections onto $m$-Symmetric Spaces}

1. We study geodesic mappings $f$ of a space $A_{n}$ with an affine connection $\nabla$ onto 2-symmetric space $\bar{A}_{n}$ with an affine connection $\bar{\nabla}$, which are characterized by the following condition [35]:

$$
\bar{R}_{i j k ; m \rho}^{h}=0,
$$

where the symbol "; " denotes a covariant derivative with respect to the connection of the space $\bar{A}_{n}$.

Since

$$
\bar{R}_{i j k m}^{h}=\frac{\partial \bar{R}_{i j k}^{h}}{\partial x^{m}}+\bar{\Gamma}_{m \alpha}^{h} \bar{R}_{i j k}^{\alpha}-\bar{\Gamma}_{m i}^{\alpha} \bar{R}_{\alpha j k}^{h}-\bar{\Gamma}_{m j}^{\alpha} \bar{R}_{i \alpha k}^{h}-\bar{\Gamma}_{m k}^{\alpha} \bar{R}_{i j \alpha}^{h}
$$

take into account (1), it follows that

$$
\bar{R}_{i j k ; m}^{h}=\bar{R}_{i j k, m}^{h}+P_{m \alpha}^{h} \bar{R}_{i j k}^{\alpha}-P_{m i}^{\alpha} \bar{R}_{\alpha j k}^{h}-P_{m j}^{\alpha} \bar{R}_{i \alpha k}^{h}-P_{m k}^{\alpha} \bar{R}_{i j \alpha}^{h} .
$$

From Equations (2) and (9), we get

$$
\bar{R}_{i j k ; m}^{h}=\bar{R}_{i j k, m}^{h}+\delta_{m}^{h} \psi_{\alpha} \bar{R}_{i j k}^{\alpha}-2 \psi_{m} \bar{R}_{i j k}^{h}-\psi_{j} \bar{R}_{i m k}^{h}-\psi_{k} \bar{R}_{i j m}^{h}-\psi_{i} \bar{R}_{m j k}^{h} .
$$

Let us differentiate (10) with respect to $x^{\rho}$ in the space $A_{n}$. Taking into account (6), we get

$$
\left(\bar{R}_{i j k ; m}^{h}\right)_{, \rho}=\bar{R}_{i j k, m \rho}^{h}+\delta_{m}^{h} \psi_{\alpha} \bar{R}_{i j k, \rho}^{\alpha}-2 \psi_{m} \bar{R}_{i j k, \rho}^{h}-\psi_{j} \bar{R}_{i m k, \rho}^{h}-\psi_{k} \bar{R}_{i j m, \rho}^{h}-\psi_{i} \bar{R}_{m j k, \rho}^{h}+B_{i j k m \rho}^{h}
$$

where

$$
\begin{aligned}
B_{i j k m \rho}^{h}= & \delta_{m}^{h} \bar{R}_{i j k}^{\alpha}\left(\frac{1}{n^{2}-1}\left(n \bar{R}_{\alpha \rho}+\bar{R}_{\rho \alpha}-\left(n R_{\alpha \rho}+R_{\rho \alpha}\right)\right)+\psi_{\alpha} \psi_{\rho}\right) \\
& -2 \bar{R}_{i j k}^{h}\left(\frac{1}{n^{2}-1}\left(n \bar{R}_{m \rho}+\bar{R}_{\rho m}-\left(n R_{m \rho}+R_{\rho m}\right)\right)+\psi_{m} \psi_{\rho}\right) \\
& -\bar{R}_{i m k}^{h}\left(\frac{1}{n^{2}-1}\left(n \bar{R}_{j \rho}+\bar{R}_{\rho j}-\left(n R_{j \rho}+R_{\rho j}\right)\right)+\psi_{j} \psi_{\rho}\right) \\
& -\bar{R}_{i j m}^{h}\left(\frac{1}{n^{2}-1}\left(n \bar{R}_{k \rho}+\bar{R}_{\rho k}-\left(n R_{k \rho}+R_{\rho k}\right)\right)+\psi_{k} \psi_{\rho}\right) \\
& -\bar{R}_{m j k}^{h}\left(\frac{1}{n^{2}-1}\left(n \bar{R}_{i \rho}+\bar{R}_{\rho i}-\left(n R_{i \rho}+R_{\rho i}\right)\right)+\psi_{i} \psi_{\rho}\right) .
\end{aligned}
$$

From the definition of covariant derivative in consequence of (1), we find

$$
\left(\bar{R}_{i j k ; m}^{h}\right)_{, \rho}=\bar{R}_{i j k ; m \rho}^{h}-P_{\alpha \rho}^{h} \bar{R}_{i j k ; m}^{\alpha}+P_{i \rho}^{\alpha} \bar{R}_{\alpha j k ; m}^{h}+P_{j \rho}^{\alpha} \bar{R}_{i \alpha k ; m}^{h}+P_{k \rho}^{\alpha} \bar{R}_{i j \alpha ; m}^{h}+P_{m \rho}^{\alpha} \bar{R}_{i j k ; \alpha}^{h} .
$$


Transforming (12) and taking into account (2) and (5), we get

$$
\left(\bar{R}_{i j k ; m}^{h}\right)_{, \rho}=\bar{R}_{i j k ; m \rho}^{h}+C_{i j k m \rho}^{h} .
$$

where

$$
\begin{aligned}
C_{i j k m \rho}^{h}= & -\delta_{\rho}^{h} \psi_{\alpha}\left(\bar{R}_{i j k, m}^{\alpha}+\delta_{m}^{\alpha} \psi_{\beta} \bar{R}_{i j k}^{\beta}-2 \psi_{m} \bar{R}_{i j k}^{\alpha}-\psi_{j} \bar{R}_{i m k}^{\alpha}-\psi_{k} \bar{R}_{i j m}^{\alpha}-\psi_{i} \bar{R}_{m j k}^{\alpha}\right) \\
& +3 \psi_{\rho}\left(\bar{R}_{i j k, m}^{h}+\delta_{m}^{h} \psi_{\alpha} \bar{R}_{i j k}^{\alpha}-2 \psi_{m} \bar{R}_{i j k}^{h}-\psi_{j} \bar{R}_{i m k}^{h}-\psi_{k} \bar{R}_{i j m}^{h}-\psi_{i} \bar{R}_{m j k}^{h}\right) \\
& +\psi_{i}\left(\bar{R}_{\rho j k, m}^{h}+\delta_{m}^{h} \psi_{\alpha} \bar{R}_{\rho j k}^{\alpha}-2 \psi_{m} \bar{R}_{\rho j k}^{h}-\psi_{j} \bar{R}_{\rho m k}^{h}-\psi_{k} \bar{R}_{\rho j m}^{h}-\psi_{\rho} \bar{R}_{m j k}^{h}\right) \\
& +\psi_{j}\left(\bar{R}_{i \rho k, m}^{h}+\delta_{m}^{h} \psi_{\alpha} \bar{R}_{i \rho k}^{\alpha}-2 \psi_{m} \bar{R}_{i \rho k}^{h}-\psi_{\rho} \bar{R}_{i m k}^{h}-\psi_{k} \bar{R}_{i \rho k}^{h}-\psi_{i} \bar{R}_{m \rho k}^{h}\right) \\
& +\psi_{k}\left(\bar{R}_{i j \rho, m}^{h}+\delta_{m}^{h} \psi_{\alpha} \bar{R}_{i j \rho}^{\alpha}-2 \psi_{m} \bar{R}_{i j \rho}^{h}-\psi_{j} \bar{R}_{i m \rho}^{h}-\psi_{\rho} \bar{R}_{i j m}^{h}-\psi_{i} \bar{R}_{m j \rho}^{h}\right) \\
& +\psi_{m}\left(\bar{R}_{i j k, \rho}^{h}+\delta_{\rho}^{h} \psi_{\alpha} \bar{R}_{i j k}^{\alpha}-2 \psi_{\rho} \bar{R}_{i j k}^{h}-\psi_{j} \bar{R}_{i \rho k}^{h}-\psi_{k} \bar{R}_{i j \rho}^{h}-\psi_{i} \bar{R}_{m j k}^{h}\right) .
\end{aligned}
$$

Let us introduce a tensor $\bar{R}_{i j k m}^{h}$ defined by

$$
\bar{R}_{i j k, m}^{h}=\bar{R}_{i j k m}^{h} .
$$

In this case, we suppose that in (14) covariant derivatives of the tensor $\bar{R}_{i j k}^{h}$ with respect to the connection of the space $A_{n}$ are expressed according to (15).

From (11) and (13), we get

$$
\bar{R}_{i j k ; m \rho}^{h}=\bar{R}_{i j k, m \rho}^{h}+\delta_{m}^{h} \psi_{\alpha} \bar{R}_{i j k, \rho}^{\alpha}-2 \psi_{m} \bar{R}_{i j k, \rho}^{h}-\psi_{j} \bar{R}_{i m k, \rho}^{h}-\psi_{k} \bar{R}_{i j m, \rho}^{h}-\psi_{i} \bar{R}_{m j k, \rho}^{h}+B_{i j k m \rho}^{h}-C_{i j k m \rho}^{h} .
$$

Let us assume that the space $\bar{A}_{n}$ is 2-symmetric. Hence, from (16), take into account (8) and (15), we find

$$
\bar{R}_{i j k, m \rho}^{h}=2 \psi_{m} \bar{R}_{i j k \rho}^{h}+\psi_{j} \bar{R}_{i m k \rho}^{h}+\psi_{k} \bar{R}_{i j m \rho}^{h}+\psi_{i} \bar{R}_{m j k \rho}^{h}-\delta_{m}^{h} \psi_{\alpha} \bar{R}_{i j k \rho}^{\alpha}-B_{i j k m \rho}^{h}+C_{i j k m \rho}^{h} .
$$

Obviously, in the space $A_{n}$, Equations (6), (15) and (17) form a closed mixed system of PDE's of Cauchy type with respect to functions $\psi_{i}(x), \bar{R}_{i j k}^{h}(x)$ and $\bar{R}_{i j k m}^{h}(x)$. The functions $\bar{R}_{i j k}^{h}(x)$ and $\bar{R}_{i j k m}^{h}(x)$ must satisfy the algebraic conditions (Ricci and Bianchi identities):

$$
\bar{R}_{i(j k)}^{h}=\bar{R}_{(i j k)}^{h}=0 \text {, and also } \bar{R}_{i(j k) m}^{h}=\bar{R}_{(i j k) m}^{h}=0 .
$$

Hence, we have given the proof.

Theorem 1. A space $A_{n}$ with an affine connection admits a geodesic mapping onto a 2-symmetric space $\bar{A}_{n}$ if and only if the mixed system of differential equations of Cauchy type in covariant derivatives (6), (15), (17) and (18) has a solution with respect to the functions $\psi_{i}(x), \bar{R}_{i j k}^{h}(x)$, and $\bar{R}_{i j k m}^{h}(x)$.

Obviously, the number of components of $\psi_{i}(x), \bar{R}_{i j k}^{h}(x), \bar{R}_{i j k m}^{h}(x)$ is $n+n^{4}+n^{5}$. Therefore, a general solution of Cauchy type system (6), (15), (17) and (18) depends on the initial conditions of these components at some point $x_{0}$. This means that the solution depends on a finite number of essential parameters. However, from conditions (19), this number of parameters is reduced, and even more so when we take into account the integrability conditions. Estimation of the parameters is in the following corollary.

Corollary 1. The general solution of the mixed system of Cauchy type (6), (15), (17) and (18) depends on no more than $n+\frac{1}{2} n(n+1)(n-1)^{2}$ essential parameters. 
2. Now, we study geodesic mapping of space $A_{n}$ onto 3-symmetric space $\bar{A}_{n}$, which are characterized by the following conditions [35]:

$$
\bar{R}_{i j k ; m \rho l}^{h}=0 .
$$

Let us covariantly differentiate (16) with respect to $x^{l}$ in the space $A_{n}$ and on the left-hand side express the covariant derivative with respect to the connection of $A_{n}$ in terms of the covariant derivative with respect to the connection of $\bar{A}_{n}$, using the formula

$$
\left(\bar{R}_{i j k ; m \rho}^{h}\right)_{, l}=\bar{R}_{i j k ; m \rho l}^{h}-P_{\alpha l}^{h} \bar{R}_{i j k ; m \rho}^{\alpha}+P_{i l}^{\alpha} \bar{R}_{\alpha j k ; m \rho}^{h}+P_{j l}^{\alpha} \bar{R}_{i \alpha k ; m \rho}^{h}+P_{k l}^{\alpha} \bar{R}_{i j \alpha ; m \rho}^{h}+P_{m l}^{\alpha} \bar{R}_{i j k ; \alpha \rho}^{h}+P_{\rho l}^{\alpha} \bar{R}_{i j k ; m \alpha}^{h} .
$$

Let tensor $\bar{R}_{i j k m \rho}^{h}$ be defined by

$$
\bar{R}_{i j k m, \rho}^{h}=\bar{R}_{i j k m \rho}^{h} .
$$

Let us assume that the space $\bar{A}_{n}$ is 3-symmetric. Hence, from the obtained equation because of (19), using substitutions and transformations, we find

$$
\bar{R}_{i j k m \rho, l}^{h}=\theta_{i j k m \rho l}^{h}
$$

where $\theta_{i j k m \rho l}^{h}$ is some tensor depending on unknown tensors $\psi_{i}, \bar{R}_{i j k}^{h}, \bar{R}_{i j k m^{\prime}}^{h} \bar{R}_{i j k m \rho^{\prime}}^{h}$ and on some tensors, which are assumed to be known.

Obviously, in the space $A_{n}$, Equations (6), (15), (20) and (21) form a closed mixed system of PDE's of Cauchy type with respect to functions $\psi_{i}(x), \bar{R}_{i j k}^{h}(x), \bar{R}_{i j k m}^{h}(x)$ and $\bar{R}_{i j k m \rho}^{h}(x)$. In addition, the algebraic conditions (18) have to be satisfied.

Hence, we have proved

Theorem 2. A space $A_{n}$ with an affine connection admits a geodesic mapping onto a 3-symmetric space $\bar{A}_{n}$ if and only if the mixed system of differential equations of Cauchy type in covariant derivatives (6), (15), (20), (21) and (18) has a solution with respect to the functions $\psi_{i}(x), \bar{R}_{i j k}^{h}(x), \bar{R}_{i j k m}^{h}(x)$ and $\bar{R}_{i j k m \rho}^{h}(x)$.

The following parameters estimation follows from the Ricci identity of curvature tensor and its derivatives.

Corollary 2. The general solution of the mixed system of Cauchy type (6), (15), (20), (21) and (18) depends on no more than $n+\frac{1}{3} n\left(n^{2}+n+1\right)\left(n^{2}-1\right)$ essential parameters.

3. Finally, we study geodesic mappings of space $A_{n}$ onto m-symmetric space $\bar{A}_{n}$, which are characterized by the following condition [35]:

$$
\bar{R}_{i j k ; \rho_{1} \rho_{2} \ldots \rho_{m}}^{h}=0 .
$$

Let us differentiate (21) covariantly $(m-2)$ times with respect to the connection of the space $A_{n}$ and on the left-hand side express the covariant derivative with respect to the connection of $A_{n}$ in terms of the covariant derivative with respect to the connection of $\bar{A}_{n}$, using the formula

$$
\begin{aligned}
\left(\bar{R}_{i j k ; \rho_{1} \ldots \rho_{\tau-2} \rho_{\tau-1}}^{h}\right)_{, \rho_{\tau}}= & \bar{R}_{i j k ; \rho_{1} \ldots \rho_{\tau-2} \rho_{\tau-1} \rho_{\tau}}^{h}-P_{\alpha \rho_{\tau}}^{h} \bar{R}_{i j k ; \rho_{1} \ldots \rho_{\tau-2} \rho_{\tau-1}}^{\alpha}+P_{i \rho_{\tau}}^{\alpha} \bar{R}_{\alpha j k ; \rho_{1} \ldots \rho_{\tau-2} \rho_{\tau-1}}^{h} \\
& +P_{j \rho_{\tau}}^{\alpha} \bar{R}_{i \alpha k ; \rho_{1} \ldots \rho_{\tau-2} \rho_{\tau-1}}^{h}+P_{k \rho_{\tau}}^{\alpha} \bar{R}_{i j \alpha ; \rho_{1} \ldots \rho_{\tau-2} \rho_{\tau-1}}^{h} \\
& +P_{\rho_{1} \rho_{\tau}}^{\alpha} \bar{R}_{i j k ; \alpha \ldots \rho_{\tau-2} \rho_{\tau-1}}^{h}+\cdots+P_{\rho_{\tau-1} \rho_{\tau}}^{\alpha} \bar{R}_{i j k ; \rho_{1} \ldots \rho_{\tau-2} \alpha}^{h} .
\end{aligned}
$$

The formula holds because of (1). 
Let us introduce a tensor $\bar{R}_{i j k \rho_{1} \rho_{2} \rho_{3}}^{h}, \ldots, \bar{R}_{i j k \rho_{1} \rho_{2} \rho_{3} \ldots \rho_{m-2} \rho_{m-1}}^{h}$ as follows

$$
\begin{aligned}
\bar{R}_{i j k \rho_{1} \rho_{2}, \rho_{3}}^{h} & =\bar{R}_{i j k \rho_{1} \rho_{2} \rho_{3}{ }^{\prime}}^{h} \cdot . \cdot \cdot \cdot \cdot \cdot \cdot \cdot \cdot \\
\bar{R}_{i j k \rho_{1} \rho_{2} \rho_{3} \ldots \rho_{m-2,} \rho_{m-1}}^{h} & =\bar{R}_{i j k \rho_{1} \rho_{2} \rho_{3} \ldots \rho_{m-2} \rho_{m-1}}^{h}
\end{aligned} .
$$

Let us assume that the space $\bar{A}_{n}$ is $m$-symmetric $(m>3)$. Hence, from the obtained equation because of (22), using substitutions and transformations, taking account of (15), (20), (23), we get

$$
\bar{R}_{i j k \rho_{1} \ldots \rho_{m-2} \rho_{m-1}, \rho_{m}}^{h}=\theta_{i j k \rho_{1} \ldots \rho_{m-1} \rho_{m}}^{h},
$$

where $\theta_{i j k \rho_{1} \ldots \rho_{m-1} \rho_{m}}^{h}$ is some tensor depending on unknown tensors $\psi_{i}, \bar{R}_{i j k^{\prime}}^{h}, \bar{R}_{i j k \rho_{1}}^{h}, \ldots, \bar{R}_{i j k \rho_{1} \ldots \rho_{m-1}}^{h}$, and on some tensors, which are assumed to be known.

Obviously, in the space $A_{n}$ the Equations (6), (15), (20), (23), (24) form a closed mixed system of PDE's of Cauchy type with respect to functions $\psi_{i}(x), \bar{R}_{i j k}^{h}(x), \bar{R}_{i j k \rho_{1}}^{h}(x), \ldots, \bar{R}_{i j k \rho_{1} \ldots \rho_{m-1}}^{h}(x)$. In addition, the algebraic conditions (18) have to be satisfied.

Hence, we have given the proof.

Theorem 3. A space $A_{n}$ with an affine connection admits a geodesic mapping onto a m-symmetric space $\bar{A}_{n}$ if and only if the mixed system of differential equations of Cauchy type in covariant derivatives (6), (15), (18), (20), (23) and (24) has a solution with respect to the functions $\psi_{i}(x), \bar{R}_{i j k}^{h}(x), \bar{R}_{i j k \rho_{1}}^{h}(x), \ldots, \bar{R}_{i j k \rho_{1} \ldots \rho_{m-1}}^{h}(x)$.

Corollary 3. The general solution of the mixed system of Cauchy type (6), (15), (18), (20), (23) and (24) depends on no more than $n+\frac{1}{3} n\left(n^{2}-1\right)\left(1+n+n^{2}+\cdots+n^{m-1}\right)$ essential parameters.

\section{Geodesic Mappings of Spaces with Affine Connections onto $m$-Ricci-Symmetric Spaces}

1. Here, we study geodesic mappings of space $A_{n}$ onto 2-Ricci-symmetric space $\bar{A}_{n}$, which are characterized by the following condition:

$$
\bar{R}_{i j ; k m}=0
$$

where $\bar{R}_{i j}$ is the Ricci tensor of $\bar{A}_{n}$.

Let us contract the Equation (16) for $h$ and $k$. Because of expressions for the tensors $B_{i j k m \rho}^{h}$ and $C_{i j k m \rho}^{h}$ we find

$$
\begin{aligned}
\bar{R}_{i j ; m \rho}= & \bar{R}_{i j, m \rho}-3 \bar{R}_{i j,(\rho} \psi_{m)}+3 \psi_{i} \psi_{(\rho} \bar{R}_{m) j}+6 \psi_{\rho} \psi_{m} \bar{R}_{i j}+3 \psi_{j} \bar{R}_{i(\rho} \psi_{m)}-\psi_{i} \bar{R}_{(m|j|, \rho)} \\
& -\psi_{j} \bar{R}_{i(\rho, m)}+\psi_{i} \psi_{j} \bar{R}_{(m \rho)}-\frac{1}{n^{2}-1}\left(2 \bar{R}_{i j}\left(n \bar{R}_{m \rho}+\bar{R}_{\rho m}-n R_{m \rho}-R_{m \rho}\right)\right. \\
& \left.+\bar{R}_{m j}\left(n \bar{R}_{i \rho}+\bar{R}_{\rho i}-n R_{i \rho}-R_{\rho i}\right)+\bar{R}_{i m}\left(n \bar{R}_{j \rho}+\bar{R}_{\rho j}-n R_{j \rho}-R_{\rho j}\right)\right),
\end{aligned}
$$

where $(i j)$ denotes an operation called symmetrization without division with respect to the indices $i$ and $j$.

Let us introduce a tensor $\bar{R}_{i j m}$

$$
\bar{R}_{i j, m}=\bar{R}_{i j m}
$$


Let us assume that space $\bar{A}_{n}$ is 2-symmetric. Hence, from (26), take into account (25) and (27), we find

$$
\begin{aligned}
\bar{R}_{i j m, k}= & 3 \bar{R}_{i j(k} \psi_{m)}-3 \psi_{i} \psi_{(k} \bar{R}_{m) j}-6 \psi_{k} \psi_{m} \bar{R}_{i j}-3 \psi_{j} \bar{R}_{i(k} \psi_{m)}+\psi_{i} \bar{R}_{(m|j| k)} \\
& +\psi_{j} \bar{R}_{i(k m)}-\psi_{i} \psi_{j} \bar{R}_{(m k)}+\frac{1}{n^{2}-1}\left(2 \bar{R}_{i j}\left(n \bar{R}_{m k}+\bar{R}_{k m}-n R_{m k}-R_{k m}\right)\right. \\
& \left.+\bar{R}_{m j}\left(n \bar{R}_{i k}+\bar{R}_{k i}-n R_{i k}-R_{k i}\right)+\bar{R}_{i m}\left(n \bar{R}_{j k}+\bar{R}_{k j}-n R_{j k}-R_{k j}\right)\right) .
\end{aligned}
$$

Obviously, in the space $A_{n}$, Equations (6), (27) and (28) form a closed system of PDE's of Cauchy type with respect to functions $\psi_{i}(x), \bar{R}_{i j}(x)$, and $\bar{R}_{i j k}(x)$.

Hence, we have given the proof.

Theorem 4. A space $A_{n}$ with an affine connection admits a geodesic mapping onto a 2-Ricci-symmetric space $\bar{A}_{n}$ if and only if the closed system of differential equations of Cauchy type in covariant derivatives (6), (27) and (28) has a solution with respect to the functions $\psi_{i}(x), \bar{R}_{i j}(x)$, and $\bar{R}_{i j k}(x)$.

Systems (6), (27) and (28) have no more than one solution for initial conditions of components $\psi_{i}(x), \bar{R}_{i j}(x), \bar{R}_{i j k}(x)$ at some point $x_{0}$. The number of parameters of $\psi_{i}\left(x_{0}\right), \bar{R}_{i j}\left(x_{0}\right)$ and $\bar{R}_{i j k}\left(x_{0}\right)$ are $n+n^{2}+n^{3}$. Therefore, the following corollary holds.

Corollary 4. The general solution of the system of Cauchy type (6), (27) and (28) depends on no more than $n+n^{2}+n^{3}$ essential parameters.

2. Now, we study geodesic mappings of space $A_{n}$ onto 3-Ricci-symmetric space $\bar{V}_{n}$, which are characterized by the condition:

$$
\bar{R}_{i j ; k m l}=0 .
$$

Let us covariantly differentiate (26) with respect to $x^{l}$ in the space $A_{n}$ and on the left-hand side express the covariant derivative with respect to the connection of $A_{n}$ in terms of the covariant derivative with respect to the connection of $\bar{A}_{n}$, using the formula

$$
\left(\bar{R}_{i j ; m k}\right)_{, l}=\bar{R}_{i j ; m k l}+P_{i l}^{\alpha} \bar{R}_{\alpha j ; m k}+P_{j l}^{\alpha} \bar{R}_{i \alpha ; m k}+P_{m l}^{\alpha} \bar{R}_{i j ; \alpha k}+P_{k l}^{\alpha} \bar{R}_{i j ; m \alpha} .
$$

Using the formulas for transition from the covariant derivatives with respect to the connection of the space $\bar{A}_{n}$ to the the covariant derivatives with respect to the connection of the space $A_{n}$, we find

$$
\bar{R}_{i j ; m k l}=\bar{R}_{i j, m k l}+\Omega_{i j m k l},
$$

where $\Omega_{i j m k l}$ is some tensor, which depends on unknown tensors $\psi_{i}, \bar{R}_{i j}, \bar{R}_{i j, k}, \bar{R}_{i j, k m}$ and, on some tensors, which are assumed to be known.

Let us introduce a tensor $\bar{R}_{i j m k}$ defined by

$$
\bar{R}_{i j m, k}=\bar{R}_{i j m k} .
$$

Let us assume that the space $\bar{A}_{n}$ is 3-symmetric. Hence, from (30), taking into account (27) and (31), we find

$$
\bar{R}_{i j m k, l}=-\Omega_{i j m k l},
$$

where the tensor $\Omega_{i j m k l}$ depends on the unknown tensors $\psi_{i}, \bar{R}_{i j}, \bar{R}_{i j k}$, and $\bar{R}_{i j k m}$.

Obviously, in the space $A_{n}$, Equations (6), (27), (31) and (32) form a closed system of PDE's of Cauchy type with respect to functions $\psi_{i}(x), \bar{R}_{i j}(x), \bar{R}_{i j k}(x)$ and $\bar{R}_{i j k m}(x)$.

Hence, we have proved 
Theorem 5. A space $A_{n}$ with an affine connection admits a geodesic mapping onto a 3-Ricci-symmetric space $\bar{A}_{n}$ if and only if the system of differential equations of Cauchy type in covariant derivatives (6), (27), (31) and (32) has a solution with respect to the functions $\psi_{i}(x), \bar{R}_{i j}(x), \bar{R}_{i j k}(x)$, and $\bar{R}_{i j k m}(x)$.

Corollary 5. The general solution of the system of Cauchy type (6), (27), (31) and (32) depends on no more than $n+n^{2}+n^{3}+n^{4}$ essential parameters.

3. Finally, we study geodesic mapping of space $V_{n}$ onto $m$-Ricci-symmetric space $\bar{V}_{n}$, which are characterized by the condition:

$$
\bar{R}_{i j ; \rho_{1} \rho_{2} \ldots \rho_{m}}=0 \text {. }
$$

It is essentially that 2- and 3-Ricci-symmetric spaces are particular cases of m-Ricci-symmetric spaces.

Let us differentiate (32) covariantly $(m-2)$ times with respect to the connection of the space $A_{n}$ and on the left-hand side express the covariant derivative with respect to the connection of $A_{n}$ in terms of the covariant derivative with respect to the connection of $\bar{A}_{n}$, using the formula

$$
\begin{aligned}
\left(\bar{R}_{i j ; \rho_{1} \ldots \rho_{\tau-2} \rho_{\tau-1}}\right)_{, \rho_{\tau}}= & \bar{R}_{i j ; \rho_{1} \ldots \rho_{\tau-2} \rho_{\tau-1} \rho_{\tau}}+P_{i \rho_{\tau}}^{\alpha} \bar{R}_{\alpha j ; \rho_{1} \ldots \rho_{\tau-2} \rho_{\tau-1}}+P_{j \rho_{\tau}}^{\alpha} \bar{R}_{i \alpha ; \rho_{1} \ldots \rho_{\tau-2} \rho_{\tau-1}} \\
& +P_{\rho_{1} \rho_{\tau}}^{\alpha} \bar{R}_{i j ; \alpha \ldots \rho_{\tau-2} \rho_{\tau-1}}+\cdots+P_{\tau-1 \rho_{\tau}}^{\alpha} \bar{R}_{i j ; \rho_{1} \ldots \rho_{\tau-2} \alpha} .
\end{aligned}
$$

Let us introduce tensors $\bar{R}_{i j k \rho_{1} \rho_{2} \rho_{3}}, \ldots, \bar{R}_{i j \rho_{1} \rho_{2} \rho_{3} \ldots \rho_{m-2} \rho_{m-1}}$ as follows:

$$
\begin{aligned}
\bar{R}_{i j \rho_{1} \rho_{2}, \rho_{3}} & =\bar{R}_{i j k \rho_{1} \rho_{2} \rho_{3},} \\
. . . & . . . \\
\bar{R}_{i j \rho_{1} \rho_{2} \ldots \rho_{m-2}, \rho_{m-1}} & =\bar{R}_{i j \rho_{1} \rho_{2} \rho_{3} \ldots \rho_{m-2} \rho_{m-1}} .
\end{aligned}
$$

Let us assume that the space $\bar{A}_{n}$ is $m$-Ricci-symmetric $(m>2)$. Hence, from the obtained equation because of (33), using substitutions and transformations, taking into account (27), (31) and (34), we get

$$
\bar{R}_{i j \rho_{1} \ldots \rho_{m-2} \rho_{m-1}, \rho_{m}}=\theta_{i j \rho_{1} \ldots \rho_{m-1} \rho_{m}}
$$

where $\theta_{i j \rho_{1} \ldots \rho_{m-1} \rho_{m}}$ is some tensor depending on unknown tensors $\psi_{i}, \bar{R}_{i j}, \ldots, \bar{R}_{i j \rho_{1}}, \bar{R}_{i j \rho_{1} \ldots \rho_{m-1}}$, and, on some tensors, which are assumed to be known.

Obviously, in the space $A_{n}$, Equations (6), (27), (31), (34) and (35) form a closed system of PDE's of Cauchy type with respect to the functions $\psi_{i}(x), \bar{R}_{i j}(x), \bar{R}_{i j \rho_{1}}(x), \ldots, \bar{R}_{i j \rho_{1} \ldots \rho_{m-1}}(x)$.

Hence, we have given the proof.

Theorem 6. A space $A_{n}$ with an affine connection admits a geodesic mapping onto a $m$-symmetric space $\bar{A}_{n}$ if and only if the system of differential equations of Cauchy type in covariant derivatives (6), (27), (31), (34) and (35) has a solution with respect to the functions $\psi_{i}(x), \bar{R}_{i j}(x), \bar{R}_{i j \rho_{1}}(x), \ldots, \bar{R}_{i j \rho_{1} \ldots \rho_{m-1}}(x)$.

Corollary 6. The general solution of the closed system of Cauchy type (6), (27), (31), (34) and (35) depends on no more than $n+n^{2}+\ldots+n^{m+1}$ essential parameters.

\section{Conclusions}

The above new results are the generalization of analogical results for geodesic mappings onto symmetric and Ricci-symmetric spaces and for the case of $m$-symmetric and $m$-Ricci symmetric spaces $(m>1)$. The generalization of $m$-recurrent, $m$-Ricci-recurrent and also $D_{n}^{m}$ and $\operatorname{Ric} D_{n}^{m}$ studied by Kaigorodov probably does not exist in the general case. 
Author Contributions: Investigation, V.B., Y.C., I.H. and P.P. All authors contributed equally and significantly in writing this article. All authors have read and agreed to the published version of the manuscript.

Funding: This research received no external funding.

Acknowledgments: The paper was supported by the project IGA_PrF_2020_014 Palacky University Olomouc and No. LO1408 "AdMaS UP" Advanced Materials, Structures and Technologies supported by Ministry of Education, Youth and Sports under the "National Sustainability Programme I" of the Brno University of Technology.

Conflicts of Interest: The authors declare no conflict of interest.

\section{References}

1. Levi-Civita, T. Sulle trasformazioni dello equazioni dinamiche. Ann. di Mat. 1896, 24, 252-300.

2. Thomas, T.Y. On projective and equiprojective geometries of paths. Proc. Natl. Acad. Sci. USA 1925, 11, 198-203.

3. Thomas, J.M. Asymmetric displacement of a vector. Trans. Am. Math. Soc. 1926, 28, 658-670.

4. Weyl, H. Zur Infinitesimalgeometrie Einordnung der projektiven und der konformen Auffassung. Göttinger Nachrichten 1921, 1921, 99-112.

5. Shirokov, P.A. Selected Investigations on Geometry; Kazan University Press: Kazan, Russia, 1966.

6. Solodovnikov, A.S. Projective transformation of Riemannian spaces. Usp. Mat. Nauk 1956, 11, 45-116.

7. Sinyukov, N.S. On geodesic mappings of Riemannian spaces onto symmetric Riemannian spaces. Dokl. Akad. Nauk SSSR 1954, 98, 21-23.

8. Sinyukov, N.S. On the theory of a geodesic mapping of Riemannian spaces. Dokl. Akad. Nauk SSSR 1966, 169, 770-772.

9. Sinyukov, N.S. Geodesic Mappings of Riemannian Spaces; Nauka: Moscow, Russia, 1979.

10. Petrov, A.Z. New Methods in the General Theory of Relativity; Nauka: Moscow, Russia, 1966.

11. Aminova, A.V. Pseudo-Riemannian manifolds with common geodesics. Russ. Math. Surv. 1993, 48, $105-160$.

12. Mikeš, J. Geodesic mappings of affine-connected and Riemannian spaces. J. Math. Sci. 1996, 78, 311-333.

13. Mikeš, J.; Vanžurová, A.; Hinterleitner, I. Geodesic Mappings and Some Generalizations; Palacky University Press: Olomouc, Czech Republic, 2009.

14. Hinterleitner, I.; Mikeš, J. On fundamental equations of geodesic mappings and their generalizations. J. Math. Sci. 2011, 174, 537-554.

15. Mikeš, J.; Stepanova, E.; Vanžurová, A.; Bácsó, S.; Berezovski, V.E.; Chepurna, O.; Chodorová, M.; Chudá, H.; Gavrilchenko, M.L.; Haddad, M.; et al. Differential Geometry of Special Mappings; Palacky University Press: Olomouc, Czech Republic, 2015.

16. Mikeš, J.; Berezovski, V.; Stepanova, E.; Chudá, H. Geodesic mappings and their generalizations. J. Math. Sci. 2016, 217, 607-623.

17. Mikeš, J.; Bácsó, S.; Berezovski, V.E.; Chepurna, O.; Chodorová, M.; Chudá, H.; Formella, S.; Gavrilchenko, M.L.; Haddad, M.; Hinterleitner, I.; et al. Differential Geometry of Special Mappings, 2nd ed.; Palacky University Press: Olomouc, Czech Republic, 2019.

18. Cartan, É. Sur une classe remarquable d'espaces de Riemann. I. Bull. S.M.F. 1926, 54, $214-264$.

19. Lichnerowicz, A. Global Theory of Connections and Holonomy Groups; Noordhoff Int. Publ. XIV: Leyden, The Netherlands, 1976; p. 250.

20. Ruse, H.S. On simply harmonic spaces. J. Lond. Math. Soc. 1946, 21, 243-247.

21. Mikeš, J. Geodesic mappings of semisymmetric Riemannian spaces. Odessk. Univ. Moscow Arch. VINITI 1976, 11, 3924-3976.

22. Mikeš, J. On geodesic mappings of 2-Ricci symmetric Riemannian spaces. Math. Notes 1980, 28, 622-624.

23. Mikeš, J. On geodesic mappings of $m$-symmetric and generally semi-symmetric spaces. Russ. Math. 1992, 36, 38-42.

24. Mikeš, J. On geodesic and holomorphic-projective mappings of generalized $m$-recurrent Riemannian spaces. Sib. Mat. Zh. 1992, 33, 215.

25. Mikeš, J. Geodesic mappings onto semisymmetric spaces. Russ. Math. 1994, 38, 35-41.

26. Fomin V.E. On geodesic mappings of infinite-dimensional Riemannian spaces onto symmetric spaces of an affine connection. Tr. Geometr. Sem. Kazan 1979, 11, 93-99.

27. Hinterleitner, I.; Mikeš, J. Geodesic mappings onto Weyl manifolds. J. Appl. Math. 2009, 2, 125-133. 
28. Sakaguchi T. On the holomorphically projective correspondence between Kählerian spaces preserwing complex structure. Hokkaido Math. J. 1974, 3, 203-212.

29. Mikeš J. Holomorphically projective mappings and their generalizations. J. Math. Sci. 1998, 89, 1334-1353.

30. Berezovskii, V.; Mikeš, J.; Peška, P. Geodesic mappings of manifolds with an affine connection onto symmetric manifolds. Geom. Integr. Quantization 2017, 18, 99-104.

31. Kaigorodov, V.R. The Riemannian spaces $D_{n}^{s}$. Structure of the curvature of spaces of type A. Soviet Math. 1974, 18, 97-105.

32. Kaigorodov, V.R. Riemannian spaces ${ }_{K}^{s}$. Tr. Geom. Sem. 1974, 5, 359-373.

33. Kaigorodov, V.R. The structure of the curvature of $D_{n}^{s}$-spaces of type B. Soviet Math. 1975, 19, 87-90.

34. Kaigorodov, V.R. The Riemannian spaces $\stackrel{*}{n}_{n}^{s}$. Second order recurrence. Soviet Math. 1975, 19, 90-92.

35. Kaigorodov, V.R. A structure of space-time curvature. J. Soviet Math. 1985, 28, 256-273.

36. Kaigorodov, V.R. Local symmetries and covariant integration for algebraically special gravitational fields. Russian Math. 1994, 38, 30-36.

37. Kowalski, O. Generalized Symmetric Spaces; Lecture Notes in Mathematics 805; Springer: Berlin/Heidelberg, Germany, 1980.

38. Mikeš, J.; Stepanov, S. On generalized semisymmetric Riemannian manifolds. AAPP, Mat. Nat. 2013, 91 , A1. doi:10.1478/AAPP.91S2A1.

39. Sobchuk, V.S. On the Ricci geodesic mapping of 4-symmetric Riemannian spaces. Sov. Math. 1991, 35, 68-69.

40. Mikeš, J.; Sobchuk, V.S. Geodesic mappings of 3-symmetric Riemannian spaces. J. Math. Sci. 1994, 69, 885-888.

41. Mikeš, J.; Radulović, Ž. On geodesic and holomorphically projective mappings of generalized recurrent spaces. Publ. Inst. Math. 1996, 59, 153-160.

42. Mikeš, J.; Radulović, Ž. On Projective Transformations of Riemannian Spaces with Harmonic Curvature. New Developments in Diff. Geom., Budapest 1996. In Proceedings of the Conference on Differential Geometry, Budapest, Hungary, 27-30 July 1996; Kluwer Acad. Publ.: Dordrecht, The Netherlands, 1999; pp. 279-283.

43. Mikeš, J.; Radulović, Ž.; Haddad, M. Geodesic and holomorphically projective mappings of $m$-pseudo- and m-quasisymmetric Riemannian spaces. Russ. Math. 1996, 40, 28-32.

44. Berezovski, V.; Hinterleitner, I.; Mikeš, J. Geodesic mappings of manifolds with an affine connection onto the Ricci symmetric manifolds. Filomat 2018, 32, 379-385.

45. Berezovski, V.; Bácsó, S.; Mikeš, J. Diffeomorphism of affine connected spaces which preserved Riemannian and Ricci curvature tensors. Miskolc Math. Notes 2017, 18, 117-124.

46. Berezovskii, V.; Mikeš, J.; Rýparová, L. Geodesic mappings of spaces with an affine connection onto generalized Ricci symmetric spaces. Filomat 2019, 33, 4475-4480.

47. Berezovski, V.; Cherevko, Y.; Rýparová, L. Conformal and geodesic mappings onto some special spaces. Mathematics 2019, 7, 664 .

48. Berezovski, V.; Mikeš, J.; Rýparová, L. Conformal and Geodesic Mappings Onto Ricci Symmetric Spaces. In Proceedings of the 19th Conference on Applied Mathematics APLIMAT 2020, Bratislava, Slovakia, 4-6 February 2020; pp. 65-72.

49. Mikeš, J. On geodesic mappings of Einstein spaces. Math. Notes 1980, 28, 922-923.

50. Mikeš, J.; Berezovski, V. Geodesic mappings of affine-connected spaces onto Riemannian spaces. In Differential Geometry and Its Applications. Proceedings of a Colloquium, Eger, Hungary, 20-25 August 1989; North-Holland Publishing Company: Amsterdam, The Netherlands, 1992; pp. 491-494.

51. Mikeš, J.; Pokorná, O.; Starko, G. Geodesic mappings between Kählerian spaces. Filomat 2002, 16, 43-50.

52. Mikeš, J.; Strambach, K. Differentiable structure on elementary geometries. Result. Math. 2009, 53, $153-172$.

53. Mikeš, J.; Strambach, K. Grünwald shift spaces. Publ. Math. 2013, 83, 85-96.

54. Mikeš, J.; Strambach, K. Shells of monotone curves. Czechoslov. Math. J. 2015, 65, 677-699.

55. Berezovskii, V.; Kovalev, L.E.; Mikeš, J. On preserving the Riemann tensor with respect to some mappings of spaces of affine connection. Russ. Math. 2018, 62, 1-6.

56. Mikeš, J.; Hinterleitner, I.; Guseva, N.I. Geodesic mappings "in large" of Ricci-flat spaces with $n$ closed geodesics. Math. Notes 2020, 108, 292-296.

57. Vesić, N.O.; Velimirović, Lj.S.; Stanković, M.S. Some invariants of equitorsion third type almost geodesic mappings. Mediterr. J. Math. 2016, 13, 4581-4590. 
58. Vesić, N.O.; Zlatanović, M.Lj.; Velimirović, A.M. Projective invariants for equitorsion geodesic mappings of semi-symmetric affine connection spaces. J. Math. Anal. Appl. 2019, 472, 1571-1580.

59. Hinterleitner, I.; Mikeš, J.; Peška, P. Fundamental equations of F-planar mappings. Lobachevskii J. Math. 2017, 38, 653-659.

(c) 2020 by the authors. Licensee MDPI, Basel, Switzerland. This article is an open access article distributed under the terms and conditions of the Creative Commons Attribution (CC BY) license (http://creativecommons.org/licenses/by/4.0/). 